\title{
A General Sudden Cosmological Singularity
}

\author{
John D. Barrow ${ }^{1}$, S. Cotsakis ${ }^{2}$ and A. Tsokaros ${ }^{3}$ \\ ${ }^{1}$ DAMTP, Centre for Mathematical Sciences, \\ Cambridge University, Cambridge CB3 0WA, UK \\ ${ }^{2,3}$ Research Group of Geometry, Dynamical Systems and Cosmology, \\ Dept. Information \& Communication Systems Engineering, \\ University of the Aegean, Karlovassi 83200, Samos, Greece.
}

September 5, 2018

\begin{abstract}
We construct an asymptotic series for a general solution of the Einstein equations near a sudden singularity. The solution is quasi isotropic and contains nine independent arbitrary functions of the space coordinates as required by the structure of the initial value problem.

PACS number: 98.80.-k
\end{abstract}

\section{Introduction}

The standard homogeneous and isotropic Friedmann-Lemaitre cosmological models of general relativity are determined by the solution of two independent Einstein equations for three unknown time-dependent functions, the metric scale factor $a(t)$, the fluid density, $\rho(t)$, or the isotropic fluid pressure, $p(t)$. If an equation of state $p=f(\rho)$ is chosen, the system closes and the two remaining unknown functions are determined. Physically 
reasonable equations of state, like those for perfect fluids, produce well-behaved expansion factors with behaviours that offer simple Newtonian interpretations. However, the challenge of providing a compelling explanation for the observed acceleration of the universe in terms of a 'dark energy' fluid has led to an exploration of other less familiar equations of state [1], motivated by the form of bulk viscous stresses [2, 3, 4], where $d p / d \rho$ is not everywhere continuous, or scenarios in which there is no equation of state at all. These less constrained material stresses introduce new possibilities for the evolution of Friedmann universes and can produce unexpected types of finite-time singularity which are far weaker than the curvature singularities that often accompany geodesic incompleteness [5, 6, 7, 8, 9, 10]. We found [11, 12, 13] a whole new class of pressure-driven singularities that keep the scale factor, $a$, the cosmological expansion rate, $\dot{a} / a$, and the density, $\rho$, finite while permitting the pressure, $p$, and the deceleration $-\ddot{a} / a$, to blow up at a finite time despite the energy conditions $\rho>0$ and $\rho+3 p>0$ holding, and regardless of the three-curvature. There is no geodesic incompleteness associated with these singularities.

The stability of general relativistic solutions with sudden singularities in the presence of small scalar, vector, and tensor perturbations has been demonstrated in a gauge covariant formalism [14], and they have also been shown to be stable against quantum particle production processes as the singularities is approached [15]. They have been investigated in a number of different cosmological setting and their behaviour has been classified in the light of other types of finite-time singularity that can arise in isotropic and anisotropic cosmologies [16, 17, 18, 19, 20, 21, 22, 23, 24, 25, 26]. The formal definitions of these singularities have been discussed by Lake [27] in the case where infinities occur in second derivatives of $a(t)$, but similar examples exist where the sudden singularity occurs in (arbitrarily) higher derivatives of $a(t)$ and so no energy conditions are threatened [13, 16]. Recently, we have also provided another formal construction procedure for these solutions in isotropic universes using fractional power series [28, 29].

Sudden singularities have appeared in a wide range of cosmological investigations 
and our aim in this paper is to try to obtain information about the general cosmological behaviour in the neighbourhood of such a singularity by an asymptotic analysis of the Einstein equations without using simplifying assumptions of homogeneity or isotropy. We shall use a function-counting method, familiar from ref [30]. Past uses of this method have failed to identify general behaviour near isotropic singularities [30, 31, 32] because of the ubiquity of chaotic behaviour near curvature singularities. However, although the full complement of independently arbitrary functions did not appear, the series expansions

obtained provided useful asymptotic forms for particular cosmological models containing fluids and combinations of fluids [33, 34]. The only use of this technique which identified a general behaviour in the Einstein cosmological equations is the study of the late-time behaviour of expanding universes with $\rho>0$ and $\rho+3 p>0$ in the presence of a positive cosmological constant. A series expansion around the de Sitter solution [35] produces a general complement of arbitrary functions in accord with the expectations of the cosmic no hair theorem [35, 36, 37, 38, 39.

In this paper we are going to seek a series expansion around the particular FRW solution with a future sudden singularity introduced in ref [11, 12]. In the limit that the sudden singularity is approached at finite future time we will determine the number of independently arbitrary functions of the three space variables that are necessary and sufficient to prescribe the solution locally on a spacelike hypersurface of constant comoving proper time.

\section{Friedmann-Lemaître sudden singularities}

Consider the most general form of sudden finite-time singularity of the sort discussed in [11, 12] that can arise in an Friedmann expanding universe. In [11] we showed that singularities of this sort can arise from a divergence of the pressure, $p$, at finite time despite the scale factor, $a(t)$, the material density, $\rho$, and the Hubble expansion rate, $H=\dot{a} / a$ remaining finite. The pressure singularity is accompanied by a divergence in 
the acceleration of the universe, $\ddot{a}$, at finite time. Remarkably, these singularities occur without violating the strong energy conditions $\rho>0$ and $\rho+3 p>0$. They can even prevent a closed Friedmann universe that obeys these energy conditions from attaining an expansion maximum [12]. In order to prevent the occurrence of these sudden singularities it is necessary to bound the pressure. A sufficient condition is to require $d p / d \rho$ to be continuous or $p / \rho$ to be finite.

We will construct a quasi-isotropic ansatz for the general behaviour by first considering the Friedmann universe with curvature parameter $k$, zero cosmological constant, and units $8 \pi G=c=1$. The Einstein equations reduce to

$$
\begin{aligned}
3 H^{2} & =\rho-\frac{3 k}{a^{2}} \\
\dot{\rho}+3 H(\rho+p) & =0, \\
\frac{\ddot{a}}{a} & =-\left(\frac{\rho+3 p}{6}\right) .
\end{aligned}
$$

In [11] we constructed an explicit example by seeking, over the time interval $0<t<t_{s}$, a solution for the scale factor $a(t)$ of the form

$$
a(t)=1+B t^{q}+C\left(t_{s}-t\right)^{n}
$$

where $B>0, q>0, C$ and $n>0$ are free constants to be determined. If we fix the zero of time by requiring $a(0)=0$, so $C t_{s}^{n}=-1$, we have

$$
a(t)=\left(\frac{t}{t_{s}}\right)^{q}\left(a_{s}-1\right)+1-\left(1-\frac{t}{t_{s}}\right)^{n},
$$

where $a_{s} \equiv a\left(t_{s}\right)$. Hence, as $t \rightarrow t_{s}$ from below, we have

$$
\ddot{a} \rightarrow q(q-1) B t^{q-2}-\frac{n(n-1)}{t_{s}^{2}\left(1-\frac{t}{t_{s}}\right)^{2-n}} \rightarrow-\infty,
$$

whenever $1<n<2$ and $0<q \leq 1$; the solution exists on the interval $0<t<t_{s}$. Hence, as $t \rightarrow t_{s}$ we have $a \rightarrow a_{s}, H \rightarrow H_{s}$ and $\rho \rightarrow \rho_{s}>0$ where $a_{s}, H_{s}$, and $\rho_{s}$ are all finite but 
$p_{s} \rightarrow \infty$. The infinite deceleration in (6) is why this sometimes also termed a 'big brake' singularity. By contrast, as $t \rightarrow 0$ there is an initial all-encompassing strong-curvature singularity, with $H \rightarrow \infty, \rho \rightarrow \infty$ and $p \rightarrow \infty$. From (6) and (3), we see that $\rho$ and $\rho+3 p$ remain positive throughout the evolution but, because $\rho$ is finite asymptotically, the dominant-energy condition, $|p| \leq \rho$, must always be violated as $t \rightarrow t_{s}$, [11, 27, 16].

Using the isotropic and homogeneous solution (15) as a guide we consider a series expansion of the metric in the neighbourhood of the sudden singularity in a general cosmological solution to the Einstein equations.

\section{General series expansions near the singularity}

\subsection{Fractional power series}

We use the Landau-Lifshitz [30] notation: Latin indices for spacetime components, Greek for spatial components. The general form of the metric in synchronous coordinates is

$$
d s^{2}=d t^{2}-\gamma_{\alpha \beta} d x^{\alpha} d x^{\beta}
$$

On approach to the sudden singularity as $t \rightarrow t_{s}$, the solution (5) has the linear asymptotic form

$$
a \rightarrow a_{s}+q\left(1-a_{s}\right)\left(1-\frac{t}{t_{s}}\right)
$$

Guided by this FRW example, (5), and its asymptotic form, (8), we carry out a change of time coordinate to place the future sudden singularity at $t=0$ and then consider a general series expansion about it, with

$$
\gamma_{\alpha \beta}=a_{\alpha \beta}+b_{\alpha \beta} t+c_{\alpha \beta} t^{n}+\ldots
$$

where $1<n<2$, and $a_{\alpha \beta}, b_{\alpha \beta}, c_{\alpha \beta}$ are functions of the space coordinates only; the inverse metric tensor is

$$
\gamma^{\alpha \beta}=a^{\alpha \beta}-b^{\alpha \beta} t-c^{\alpha \beta} t^{n}+\cdots,
$$


so that $\gamma_{\alpha \beta} \gamma^{\beta \gamma}=\delta_{\alpha}^{\gamma}$. We note that $a_{\alpha \beta} a^{\beta \gamma}=\delta_{\alpha}^{\gamma}$ and the indices of $b_{\alpha \beta}, c_{\alpha \beta}$ are raised by $a^{\alpha \beta}$. The extrinsic curvature, its derivatives, and its contractions are, to leading order,

$$
\begin{aligned}
K_{\alpha \beta} & =\frac{\partial \gamma_{\alpha \beta}}{\partial t}=b_{\alpha \beta}+c_{\alpha \beta} n t^{n-1}+\cdots, \\
K_{\beta}^{\alpha} & =b_{\beta}^{\alpha}+n c_{\beta}^{\alpha} t^{n-1}+\cdots, \\
K & =b+n c t^{n-1}+\cdots, \\
\frac{\partial K_{\beta}^{\alpha}}{\partial t} & =n(n-1) c_{\beta}^{\alpha} t^{n-2}+\cdots, \\
\frac{\partial K}{\partial t} & =n(n-1) c t^{n-2}+\cdots, \\
K_{\alpha}^{\beta} K_{\beta}^{\alpha} & =b_{\mu \nu} b^{\mu \nu}+2 n b_{\mu \nu} c^{\mu \nu} t^{n-1}+\cdots, \\
K K_{\beta}^{\alpha} & =b b_{\beta}^{\alpha}+n\left(c b_{\beta}^{\alpha}+b c_{\beta}^{\alpha}\right) t^{n-1}+\cdots
\end{aligned}
$$

The components of the Ricci tensor are

$$
\begin{aligned}
R_{0}^{0} & =-\frac{1}{2} \frac{\partial K}{\partial t}-\frac{1}{4} K_{\alpha}^{\beta} K_{\beta}^{\alpha}=-\frac{n(n-1) c}{2} t^{n-2}-\frac{1}{4} b^{\mu \nu} b_{\mu \nu}-\frac{n}{2} c^{\mu \nu} b_{\mu \nu} t^{n-1}+\cdots, \\
R_{\alpha}^{0} & =\frac{1}{2}\left(K_{\alpha ; \beta}^{\beta}-K_{; \alpha}\right)=\frac{1}{2}\left(b_{\alpha ; \beta}^{\beta}-b_{; \alpha}\right)+\frac{n}{2}\left(c_{\alpha ; \beta}^{\beta}-c_{; \alpha}\right) t^{n-1}+\cdots, \\
R_{\beta}^{\alpha} & =-Q_{\beta}^{\alpha}-\frac{1}{4} K K_{\beta}^{\alpha}-\frac{1}{2} \frac{\partial K_{\beta}^{\alpha}}{\partial t}=-\frac{n(n-1)}{2} c_{\beta}^{\alpha} t^{n-2}-\left[\frac{1}{4} b b_{\beta}^{\alpha}+P_{\beta}^{\alpha}\right]-\frac{n}{4}\left[c b_{\beta}^{\alpha}+b c_{\beta}^{\alpha}\right] t^{n-1}+\cdots,
\end{aligned}
$$

where $Q_{\alpha \beta}$ is the Ricci tensor associated with $\gamma_{\alpha \beta}$, and $P_{\alpha \beta}$ is the Ricci tensor associated with $a_{\alpha \beta}$. In the synchronous reference frame

$$
g_{00}=1, \quad g_{0 \alpha}=0 .
$$

The 4-velocity $u^{i}=\left(u^{0}, u^{\alpha}\right)$ has $u^{0}=u_{0}$ and

$$
1=u_{i} u^{i}=u_{0}^{2}-\left(a_{\alpha \beta}+b_{\alpha \beta} t+c_{\alpha \beta} t^{n}+\cdots\right) u^{\alpha} u^{\beta} .
$$

The stress energy tensor for a perfect fluid

$$
T_{j}^{i}=8 \pi\left[(\rho+p) u^{i} u_{j}-p \delta_{j}^{i}\right]
$$


gives (to lowest order)

$$
\begin{aligned}
T_{0}^{0} & =(\rho+p) u_{0}^{2}-p \approx \rho, \\
T_{\alpha}^{0} & =(\rho+p) u_{0} u_{\alpha} \approx(\rho+p) u_{\alpha}, \\
T_{\beta}^{\alpha} & =(\rho+p) u^{\alpha} u_{\beta}-p \delta_{\beta}^{\alpha} \approx-p \delta_{\beta}^{\alpha} .
\end{aligned}
$$

From the Einstein equations

$$
R_{j}^{i}-\frac{1}{2} R \delta_{j}^{i}=8 \pi T_{j}^{i}
$$

we use the $\left(\begin{array}{l}0 \\ 0\end{array}\right)$ component to calculate the energy density and the trace of the $\left(\begin{array}{l}\alpha \\ \beta\end{array}\right)$ equations to calculate the pressure. The 4 -velocity can be calculated then from the $\left(\begin{array}{l}0 \\ \alpha\end{array}\right)$ component. Also we use the $\left(\begin{array}{l}\alpha \\ \beta\end{array}\right)$ components to get restrictions on the arbitrary functions of $a_{\alpha \beta}, b_{\alpha \beta}, c_{\alpha \beta}$. We have

$$
16 \pi \rho=\frac{1}{2}\left(R_{0}^{0}-R_{\alpha}^{\alpha}\right)=\left(P+\frac{b^{2}-b^{\mu \nu} b_{\mu \nu}}{4}\right)-\frac{n}{2}\left(b^{\mu \nu} c_{\mu \nu}-b c\right) t^{n-1}+\cdots,
$$

while from the trace of the $\left(\begin{array}{l}\alpha \\ \beta\end{array}\right)$ component we get

$16 \pi p=\frac{1}{2}\left(R_{0}^{0}+\frac{1}{3} R_{\alpha}^{\alpha}\right)=-\frac{2 n(n-1) c}{3} t^{n-2}-\frac{3 b^{\mu \nu} b_{\mu \nu}+b^{2}+4 P}{12}-\frac{n}{2}\left(b^{\mu \nu} c_{\mu \nu}+\frac{b c}{3}\right) t^{n-1}+\cdots$

The Ricci scalar is

$$
R=R_{i}^{i}=-n(n-1) c t^{n-2}-\frac{b_{\mu \nu} b^{\mu \nu}+b^{2}+4 P}{4}-\frac{n}{2}\left(b^{\mu \nu} c_{\mu \nu}+b c\right) t^{n-1}+\cdots,
$$

the $\left(\begin{array}{l}\alpha \\ \beta\end{array}\right)$ components of the Einstein equations give

$$
R_{\beta}^{\alpha}=\frac{1}{2}(R-16 \pi p) \delta_{\beta}^{\alpha},
$$

and therefore

$$
\begin{aligned}
& -\frac{n(n-1)}{2} c_{\beta}^{\alpha} t^{n-2}-\left(\frac{1}{4} b b_{\beta}^{\alpha}+P_{\beta}^{\alpha}\right)-\frac{n}{4}\left(c b_{\beta}^{\alpha}+b c_{\beta}^{\alpha}\right) t^{n-1}+\cdots \\
= & -\frac{n(n-1) c}{6} \delta_{\beta}^{\alpha} t^{n-2}-\delta_{\beta}^{\alpha}\left(\frac{b^{2}+4 P}{12}\right)-\delta_{\beta}^{\alpha} \frac{n b c}{6} t^{n-1}+\cdots
\end{aligned}
$$


From the $O\left(t^{n-2}\right)$ terms, we get the constraints

$$
c_{\beta}^{\alpha}=\frac{c}{3} \delta_{\beta}^{\alpha} \quad \text { or } \quad c_{\alpha \beta}=\frac{c}{3} a_{\alpha \beta},
$$

and so the components $c_{\alpha \beta}$ are all but one determined from $a_{\alpha \beta}$.

Since $\gamma_{\alpha \beta}$ is to order $t^{n}$, the extrinsic curvature will be $O\left(t^{n-1}\right)$ and the Ricci components $R_{0}^{0}, R_{\beta}^{\alpha}$ will be $O\left(t^{n-2}\right)$. Hence this will be the only restriction on $a_{\alpha \beta}, b_{\alpha \beta}, c_{\alpha \beta}$. Therefore, we have $6+6+1=13$ independent functions. Subtracting the 4 coordinate covariances which may still be used to remove four functions, leaves 9 independent arbitrary functions of the three space coordinates on a surface of constant $t$ time.

This is the maximal number of independent arbitrary spatial functions expected in a local representation of part of the general solution of Einstein's equations near a sudden singularity. In general, we expect there will be $6 \times g_{\alpha \beta}$ and $6 \times \dot{g}_{\alpha \beta}$, plus 3 free velocity components, $u_{\alpha}$, and $p$ and $\rho$, giving a total of 17 independent functions. We can remove four of these by using the $R_{a}^{0}$ constraints and four more by using the general coordinate covariances. This leaves a total of 9 free functions expected in the general solution for the metric locally. If an equation of state had been assumed to relate the pressure to the density this number would have been reduced by 1 to 8 .

We have found that the pressure is $O\left(t^{n-2}\right)$, so $\rho+p=O\left(t^{n-2}\right)$, and from

$$
R_{\alpha}^{0}=8 \pi(\rho+p) u_{0} u_{\alpha} \approx 8 \pi(\rho+p) u_{\alpha},
$$

since $R_{\alpha}^{0} \approx O\left(t^{0}\right)$, we get that $u_{\alpha} \approx O\left(t^{-(n-2)}\right)$, and the full expansion is

$$
u_{\alpha}=-\frac{3\left(b_{\alpha ; \beta}^{\beta}-b_{; \alpha}\right)}{2 n(n-1) c} t^{2-n} \equiv \Phi_{\alpha} t^{2-n}, \quad 0<2-n<1 .
$$

The equation for the stress energy conservation $T_{; i}^{i j}=0$ is split into time and spatial components as follows:

$$
\begin{aligned}
& T_{; i}^{i 0}=T_{; \alpha}^{\alpha 0}+\partial_{t} T^{00}+\frac{1}{2}\left(K T^{00}+K_{\alpha \beta} T^{\alpha \beta}\right)=0, \\
& T_{; i}^{i \beta}=T_{; \alpha}^{\alpha \beta}+\partial_{t} T^{0 \beta}+\frac{1}{2} K T^{0 \beta}+K_{\alpha}^{\beta} T^{0 \alpha}=0,
\end{aligned}
$$


where

$$
T^{00}=\rho, \quad T^{0 \alpha}=(\rho+p) u^{\alpha}, \quad T^{\alpha \beta}=p \gamma^{\alpha \beta} .
$$

The time component gives

$$
\left[(\rho+p) u^{\alpha}\right]_{; \alpha}+\partial_{t} \rho+\frac{1}{2} K(\rho+p)=0
$$

To lowest order, the first term of eq. (10) is constant $\left(O\left(t^{0}\right)\right)$. The other two terms to lowest order are $O\left(t^{n-2}\right)$; therefore, to lowest order the equation is $O\left(t^{n-2}\right)$. Hence the $O\left(t^{n-2}\right)$ term of $\partial_{t} \rho$ must cancel the $O\left(t^{n-2}\right)$ term of $\frac{1}{2} K(\rho+p)$. The first term is

$$
16 \pi(\rho+p) u^{\alpha}=-a^{\alpha \beta}\left(b_{\beta ; \mu}^{\mu}-b_{; \beta}\right)-n a^{\alpha \beta}\left(c_{\beta ; \mu}^{\mu}-c_{; \beta}\right) t^{n-1}+\cdots
$$

The second is

$$
16 \pi \partial_{t} \rho=-\frac{n(n-1)}{2}\left(b_{\mu \nu} c^{\mu \nu}-b c\right) t^{n-2}+\cdots
$$

and the third is

$$
\frac{1}{2} K(16 \pi \rho+16 \pi p)=-\frac{n(n-1) b c}{3} t^{n-2}+\cdots .
$$

Therefore this equation will be satisfied to order $O\left(t^{n-2}\right)$ if

$$
\frac{n(n-1)}{2}\left(b_{\mu \nu} c^{\mu \nu}-b c\right)+\frac{n(n-1) b c}{3}=0,
$$

that is, by

$$
b_{\mu \nu} c^{\mu \nu}=\frac{1}{3} b c
$$

which is indeed satisfied because of the constraint eq. (9) on $c_{\alpha \beta}$ found earlier. Therefore, the time component of $T_{; i}^{i j}=0$ does not give any new constraints on the terms of the series.

Similarly, the spatial part of the stress-energy conservation is expressed as

$$
\gamma^{\alpha \beta} p_{; \alpha}+\partial_{t}\left[(\rho+p) u^{\beta}\right]+\frac{1}{2} K(\rho+p) u^{\beta}+K_{\alpha}^{\beta}(\rho+p) u^{\alpha}=0 .
$$

The lowest order of the first two terms is $O\left(t^{n-2}\right)$ while the lowest order of the last two terms is $O\left(t^{0}\right)$ and so they are neglected. For the first term,

$$
16 \pi \gamma^{\alpha \beta} p_{; \alpha}=-\frac{2 n(n-1)}{3} a^{\alpha \beta} c_{; \alpha} t^{n-2}+\cdots
$$


while for the second,

$$
16 \pi \partial_{t}\left[(\rho+p) u^{\beta}\right]=-n(n-1) a^{\beta \nu}\left(c_{\nu ; \mu}^{\mu}-c_{; \nu}\right) t^{n-2}+\cdots
$$

Hence, we must have

$$
\frac{2 n(n-1)}{3} a^{\alpha \beta} c_{; \alpha}+n(n-1) a^{\beta \nu}\left(c_{\nu ; \mu}^{\mu}-c_{; \nu}\right)=0
$$

or

$$
c_{\alpha ; \beta}^{\beta}=\frac{1}{3} c_{; \alpha}
$$

which again is satisfied by the restriction of eq. (9) on $c_{\alpha \beta}$. Hence, both the temporal and the spatial components of the conservation of the stress energy tensor are satisfied to lowest order by the constraint on $c_{\beta}^{\alpha}$ that we have found already and there remain 9 independent functions in our series solution.

As an aside we note that he speed of sound is

$$
v_{s}^{2}=\frac{d p}{d \rho}=\frac{\dot{p}}{\dot{\rho}}=\frac{4(2-n) c}{3\left(b c-b_{\alpha \beta} c^{\alpha \beta}\right)} t^{-1}
$$

which goes to infinity as $t \rightarrow 0$ which is unsurprising given the divergence of the pressure at the sudden singularity.

\subsection{Puiseux and logarithmic series}

We shall now discuss some other series expansions around the singularity. Consider first a Puiseux series of the form

$$
a(t)=\sum_{j=0}^{\infty} a_{j} t^{j}+t^{n}\left[b_{0}+t^{1 / q} b_{1}+t^{2 / q} b_{2}+\cdots\right]
$$

as an asymptotic form for the Friedmann-Lemaître equations instead of eq. (5). This suggests an ansatz for the quasi-isotropic form of the general inhomogeneous metric of the form

$$
\gamma_{\alpha \beta}=a_{\alpha \beta}+b_{\alpha \beta} t+c_{\alpha \beta} t^{n}+\psi_{\alpha \beta} t^{m}+\omega_{\alpha \beta} t^{r}+\cdots
$$


with

$$
1<n<m<r<2
$$

If we perform the same analysis we will find again that $a_{\alpha \beta}$ and $b_{\alpha \beta}$ are arbitrary and

$$
c_{\alpha \beta}=\frac{c}{3} a_{\alpha \beta}, \quad \text { and } \quad \psi_{\alpha \beta}=\frac{\psi}{3} a_{\alpha \beta}, \quad \text { and } \quad \omega_{\alpha \beta}=\frac{\omega}{3} a_{\alpha \beta},
$$

with $c, \psi, \omega$ again arbitrary. The pressure and the density will be

$$
\begin{aligned}
16 \pi \rho & =16 \pi \rho_{0}+\frac{n b c}{3} t^{n-1}+\frac{m b \psi}{3} t^{m-1}+\frac{r b \omega}{3} t^{r-1}+\cdots \\
16 \pi p & =-\frac{2 n(n-1) c}{3} t^{n-2}\left[1+\frac{m(m-1) \psi}{n(n-1) c} t^{m-n}+\frac{m(m-1) \omega}{n(n-1) c} t^{r-n}+\cdots\right]
\end{aligned}
$$

and

$$
m-n>0 \quad r-n>0 .
$$

Therefore we have the same phenomenon as with the series that was described in the previous section. If we keep adding terms of the form $t^{s}$ with $1<s<2$ we will getting one more arbitrary function so if we add an infinite number of terms we will have an infinite number of functions. The reason that they are irrelevant is that they do not alter the nature of the singularity in its neighborhood as can be seen from the density and pressure eqs. (11)-(12). In other words, all three forms

$$
\begin{aligned}
& \gamma_{\alpha \beta}=a_{\alpha \beta}+b_{\alpha \beta} t+c_{\alpha \beta} t^{n}+\cdots, \\
& \gamma_{\alpha \beta}=a_{\alpha \beta}+b_{\alpha \beta} t+c_{\alpha \beta} t^{n}+\psi_{\alpha \beta} t^{m}+\omega_{\alpha \beta} t^{r}+\cdots, \\
& \gamma_{\alpha \beta}=a_{\alpha \beta}+b_{\alpha \beta} t+c_{\alpha \beta} t^{n}+\psi_{\alpha \beta} t^{m} \ln (t) \cdots
\end{aligned}
$$

give

$$
\lim _{t \rightarrow 0} \rho=\rho_{0}, \quad \text { and } \quad 16 \pi \lim _{t \rightarrow 0} p=-\lim _{t \rightarrow 0} \frac{2 n(n-1) c}{3} t^{n-2}
$$

and hence the same behaviour on approach to the sudden singularity as $t \rightarrow 0$. Thus the number of relevant independent functions is $9=6+6+1-4$ which is the number required for a specification of the general solution. 


\section{Discussion}

If we had sought a power-series expansion of the metric with time dependence dictated by the form of the radiation-dominated Friedmann-Lemaitre universe on approach to an initial big bang curvature singularity as $t \rightarrow 0$ then it would have taken the form of eq. (7) with

$$
\gamma_{\alpha \beta}=t a_{\alpha \beta}+t^{2} b_{\alpha \beta}+\ldots
$$

with $a_{\alpha \beta}$, and $b_{\alpha \beta}$ spatial functions [30, 31, 32] However, at most 3 arbitrary spatial functions in the series expansion are left free in this solution. A general solution of this form, with a radiation equation of state requires 8 independently arbitrary functions of space. The reason for the non-generic form of (13) for the radiation fluid is its quasiisotropic character. On approach to a general relativistic cosmological singularity the anisotropic shear and 3-curvatures dominate the dynamics and destroy the quasi isotropic character of a radiation-dominated universe.

By contrast, our results are quite different. We find that on approach to a latetime sudden singularity, where the density, expansion rate, and metric remain finite, it is possible for a quasi-isotropic solution to be part of the general solution of the Einstein equations. This solution has no equation of state and is characterised by nine independently arbitrary spatial functions. On approach to a sudden singularity the mean scale factor and the shear approach constant values, the chaotically anisotropic degrees of freedom which dominate at an initial curvature singularity are frozen out, and anisotropic velocities grind to a halt because of the divergent pressure and inertia. The situation is far simpler than for a initial vacuum or $p<\rho$ dominated fluid singularity. Comparable simplicity is achieved in the non-singular late-time approach to a quasiisotropic de Sitter universe in the presence of a cosmological constant or a $p=-\rho$ fluid, shown by Starobinsky [35]. We have shown that there is an analogous 'no hair' behaviour on approach to a late-time sudden singularity and the general structure of such a finite-time singularity admits a simple quasi-isotropic form. 


\section{References}

[1] Barrow J D 1990 Phys. Lett. B 235, 40

[2] Barrow J D 1987 Phys. Lett. B 180335

[3] Barrow J D 1988 Nucl. Phys. B 310743

[4] Barrow J D 1990 in The Formation and Evolution of Cosmic Strings ed G Gibbons, S W Hawking and T Vaschaspati (Cambridge: Cambridge UP) p 449

[5] Tipler F J 1977 Phys. Lett. A 648

[6] Królak A 1986 Class. Quantum Grav. 3267

[7] Fernandez-Jambrina L and Lazkoz R 2004 Phys. Rev. D 70 121503(R)

[8] Fernandez-Jambrina L and Lazkoz R 2006 Phys. Rev. D 74064030

[9] Balcerzak A and Dąbrowski M P 2006 Phys. Rev. D 73101301

[10] Hawking S W and Ellis G F R 1973 The Large Scale Structure of Space-time (Cambridge: Cambridge University Press)

[11] Barrow J D 2004 Class. Quantum Grav. 21 L79

[12] Barrow J D Galloway G J and Tipler F J 1986 Mon. Not. R. Astron. Soc. 223835

[13] Barrow J D 2004 Class. Quantum Grav. 215619

[14] Barrow J D and Lip S Z W 2009 Phys. Rev. D 80043518

[15] Barrow J D Batista A B Fabris J C and Houndjo S 2008 Phys. Rev. D 78123508

[16] Barrow J D and Tsagas 2005 C G Class. Quantum Grav. 221563

[17] Stefancic H 2005 Phys. Rev. D 71084024 
[18] Copeland E J Sami M and Tsujikawa S 2006 Int. J. Mod. Phys. D 151753 Nojiri S and Odintsov S D 2005 Phys. Rev. 72023003 Nojiri S and Odintsov S D 2004 Phys. Lett. B 5951 Nojiri S and Odintsov S D 2004 Phys. Rev. D 103522 Gorini V, Kamenshchik A Y, Moschella U and Pasquier V 2004 Phys. Rev. D 69123512

[19] Cotsakis S and Klaoudatou I 2007 J. Geom. Phys. 571303

[20] Shtanov Y and Sahni V 2002 Class. Quantum Grav. 19 L101

[21] Dąbrowski M P 2005 Phys. Rev. D 71103505

[22] Nojiri S and Odintsov S D 2004 arXiv:hep-th/0412030

[23] Nojiri S, Odintsov S D and Tsujikawa S 2005 Phys. Rev. D 71063004

[24] Cattoen C and Visser M 2005 Class. Quantum Grav. 224913

[25] Dą̧browski M P 2005 Phys. Lett. B 625184

[26] Dąbrowski M P, Denkiewicz T and Hendry M A 2007 Phys. Rev. D 75123524

[27] Lake K 2004 Class. Quantum Grav. 21 L129

[28] Cotsakis S and Barrow J D 2007 J. Phys.: Conf. Ser. 68012004

[29] Barrow J D, Cotsakis S and Tsokaros A 2010 gr-qc/1003.1027

[30] Landau L and Lifshitz E M 1975 The Classical Theory of Fields 4th revised edn (Oxford: Pergamon) p 368

[31] Khalatnikov I M and Lifshitz E M 1961 Sov. Phys. -JETP 12108

[32] Khalatnikov I M and Lifshitz E M 1964 Sov. Phys. - Usp. 6495

[33] Khalatnikov I M, A Y Kamenshchik, M Martellini and A A Starobinsky $2003 \mathrm{~J}$. Cosmol. Astropart. Phys. JCAP03(2003)001 
[34] Khalatnikov I M , A Y Kamenshchik and A A Starobinsky 2002 Class. Quantum Grav. 193845

[35] Starobinsky A A 1983 Sov. Phys. - JETP Lett. 3766

[36] Wald R M 1983 Phys. Rev. D 282118

[37] Barrow J D1983 in The Very Early Universe, ed G Gibbons, S W Hawking and S T C Siklos, (Cambridge: Cambridge University Press) p 267

[38] Jensen L G and Stein Schabes J A 1987 Phys. Rev. D 351146

[39] Rendall A D 2004 Ann. Henri Poincaré 51041 